\title{
Cruciferous Vegetables, Isothiocyanates, and Prevention of Bladder Cancer
}

\author{
Omkara L. Veeranki • Arup Bhattacharya $\cdot$ Li Tang • \\ James R. Marshall • Yuesheng Zhang
}

Published online: 18 February 2015

(C) Springer International Publishing AG 2015

\begin{abstract}
Approximately $80 \%$ of human bladder cancers (BC) are non-muscle invasive when first diagnosed and are usually treated by transurethral tumor resection. But 50-80\% of patients experience cancer recurrence. Agents for prevention of primary $\mathrm{BC}$ have yet to be identified. Existing prophylactics against $\mathrm{BC}$ recurrence, e.g., Bacillus Calmette-Guerin (BCG), have limited efficacy and utility; they engender significant side effects and require urethral catheterization. Many cruciferous vegetables, rich sources of isothiocyanates (ITCs), are commonly consumed by humans. Many ITCs possess promising chemopreventive activities against $\mathrm{BC}$ and its recurrence. Moreover, orally ingested ITCs are selectively delivered to the bladder via urinary excretion. This review is focused on urinary delivery of ITCs to the bladder, their cellular uptake, their chemopreventive activities in preclinical and epidemiological studies that are particularly relevant to prevention of $\mathrm{BC}$ recurrence and progression, and their chemopreventive mechanisms in $\mathrm{BC}$ cells and tissues.
\end{abstract}

Keywords Bladder cancer · Isothiocyanate $\cdot$ Cruciferous vegetable $\cdot$ Chemoprevention

\section{Introduction}

Bladder cancer (BC) is currently the fourth most common malignancy in men and the twelfth most common malignancy in women, with estimated new cases of 56,390 in men and 18,300 in women and estimated deaths of 11,179 in men and 4,410 in

This article is part of the Topical Collection on Cancer Chemoprevention

O. L. Veeranki · A. Bhattacharya $\cdot$ L. Tang $\cdot$ J. R. Marshall $\cdot$ Y. Zhang $(\bowtie)$

Department of Cancer Prevention and Control, Roswell Park Cancer Institute, Elm and Carlton Streets, Buffalo, NY 14263, USA

e-mail: yuesheng.zhang@roswellpark.org women in 2014 in the USA alone [1]. Cigarette smoking (mainly exposure to aromatic amines) accounts for approximately $50 \%$ of $\mathrm{BC}$ in men and women [2]. Other important risk factors include occupational exposure to aromatic amines in aluminum, leather, rubber, textile, paint and dye industries, and exposure to arsenic-laced water [3]. $\mathrm{BC}$ originates primarily in the transitional cell epithelium (urothelial epithelium) that lines the inner surface of the bladder and is directly exposed to urine, which is also known as transitional cell carcinoma (TCC) [4]. Approximately $75 \%$ of newly diagnosed $\mathrm{BC}$ are non-muscle invasive (NMIBC), also known as superficial BC; these are typically treated with endoscopic transurethral resection (TUR), which may be followed by an intravesical therapy, depending on the stage and grade of the cancer [5]. Risk of recurrence is $50-60 \%$ for grades 1 and 2 tumors and $80 \%$ for grade 3 tumors [6, 7], with a median time to first recurrence of 2.7 years [8]. Recurrence of NMIBC occurs due to at least four reasons: incomplete resection, tumor cell reimplantation, growth of microscopic tumors, and new tumor formation [9]. Approximately $20 \%$ of NMIBC progress to muscle invasive stage $[10,11]$. Prognosis is poor once muscle invasion or distant metastasis has occurred. Given the high risk of recurrence and disease progression, careful surveillance after cancer removal by TUR via cystoscopy is currently a standard clinical practice; this makes $\mathrm{BC}$ one of the most expensive malignancies to treat per patient in the USA [12]. Intravesical therapies with $\mathrm{Ba}$ cillus Calmette-Guerin (BCG) or chemotherapeutic agents (e.g., mitomycin C), delivered via a urethral catheter, are used to prevent or delay recurrence after TUR [13]. Although BCG has been more effective than other agents, $20-40 \%$ of patients fail to respond [14]. Moreover, besides requiring transurethral manipulations for drug administration, the current preventive therapies are often associated with poor tolerance and high incidence of local adverse effects [15].

Many naturally occurring isothiocyanates (ITCs), including allyl isothiocyanate (AITC), benzyl isothiocyanate 
(BITC), erucin (ECN), phenethyl isothiocyanate (PEITC), and sulforaphane (SF) (see Table 1 for their chemical structures) have shown promising cancer chemopreventive activities [16-22]. The chemopreventive mechanisms of ITCs include modulation of cancer-related proteins involved in inflammation, cell cycle arrest, cell death, angiogenesis, invasion, and metastasis along with modulation of carcinogenmetabolizing enzymes [23]. BITC, PEITC, and SF also target cancer stem cells [24-27]. ITCs inhibit the development of carcinogen-induced cancers in rodent organs, including the

Table 1 Cruciferous vegetables, their most abundant ITCs, and conversion of glucosinolates to ITCs

Cruciferous vegetables Derived ITC Chemical structure of ITC

Cabbage, Horseradish $\quad$ AITC

$\mathrm{H}_{2} \mathrm{C}=\mathrm{HC}-\mathrm{H}_{2} \mathrm{C}-\mathrm{N}=\mathrm{C}=\mathrm{S}$

Mustard, Wasabi

Garden cress

BITC

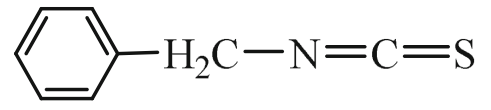

Indian cress

Broccoli

ECN

Arugula

$$
\mathrm{H}_{3} \mathrm{C}-\mathrm{S}-\left(\mathrm{H}_{2} \mathrm{C}\right)_{4}-\mathrm{N}=\mathrm{C}=\mathrm{S}
$$

Watercress

PEITC

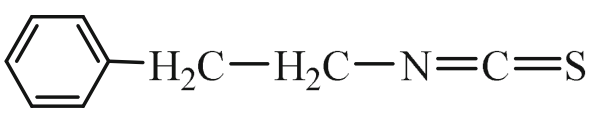

Broccoli

SF

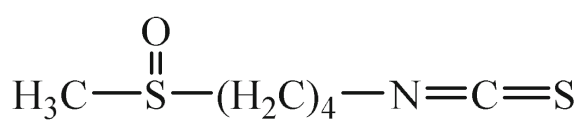

Broccoli sprouts

Myrosinase-catalyzed hydrolysis of glucosinolates<smiles>[R]C(SC1OC(CO)C(O)C(O)C1O)=[N+]([O-])O</smiles>

Glucosinolate<smiles>[R]C([S-])=NOS(=O)(=O)[O-]</smiles>

$\mathrm{HSO}_{4}^{-}$<smiles>CC(C)C#CC(C)CC(C)C</smiles>
Specifier proteins $\mathrm{R}-\mathrm{S}-\mathrm{C} \equiv \mathrm{N}$

Thiohydroximate-O-sulfonate

$$
\begin{gathered}
\mathrm{R}-\mathrm{C} \equiv \mathrm{N} \\
\text { Nitrile, } \\
\text { Epithionitrile }
\end{gathered}
$$

$\mathrm{R}-\mathrm{N}=\mathrm{C}=\mathrm{S}$

Isothiocyanate 
bladder, colon, esophagus, liver, lung, mammary gland, pancreas, skin, and small intestine [28-30]. In xenograft cancer models, AITC, BITC, PEITC, and SF inhibit BC [31, 32], breast cancer [33, 34], osteosarcoma [35], pancreatic cancer [36], or prostate cancer [37, 38]. PEITC and SF also inhibit colon cancer development induced by inactivation of the Apc tumor suppressor [39, 40].

Many lines of evidence suggest that ITCs are highly promising for prevention of $\mathrm{BC}$ and $\mathrm{BC}$ recurrence. In this review, we consider the relationship of ITCs to cruciferous vegetables, selective delivery of ITCs to the bladder, cellular uptake of ITCs, preclinical evidence of cancer chemopreventive activities and molecular mechanisms of ITCs in BC cells and tissues, epidemiologic evidence of chemopreventive activities of ITC-rich cruciferous vegetables in the bladder, and the potential adverse effects of ITCs.

\section{ITCs and Cruciferous Vegetables}

ITCs are characterized by the presence of an electrophilic functional group $-\mathrm{N}=\mathrm{C}=\mathrm{S}$ and a side chain unique to each ITC (Table 1). The chemopreventive actions of ITCs are mediated primarily by direct reaction of the carbon atom of the $-\mathrm{N}=\mathrm{C}=\mathrm{S}$ group with cellular nucleophiles, including many cancerrelated proteins [41, 42] (also see section "Molecular Mechanisms by Which Cruciferous Vegetables and ITCs Target BC Cells"). The side chains of ITCs may modulate their chemopreventive activity by influencing the electrophilicity of the $-\mathrm{N}=\mathrm{C}=\mathrm{S}$ group and the lipophilicity of the ITC $[41,43]$.

Many commonly consumed cruciferous vegetables, such as broccoli, cabbage, garden cress, horseradish, mustard seed, wasabi, and watercress are rich sources of ITCs with significant cancer preventive activities (Table 1). ITCs, stored as glucosinolates ( $\beta$-thioglucoside $N$-hydroxysulfates) in cruciferous vegetables, are generated through a myrosinasecatalyzed hydrolysis of their precursors when plant cells are damaged, first forming thiohydroximate- $O$-sulfonates (Table 1) [44]. Myrosinase, a thioglucoside glucohydrolase, is normally segregated from glucosinolates in plant cells [44]. While ITCs are products of spontaneous rearrangement of the thiohydroximate- $O$-sulfonates, nitriles, epithionitriles, and thiocyanates may form at the expense of ITCs due to the interaction of the intermediates with specifier proteins which exist in certain vegetables $[45,46]$. Mild heating of vegetables $\left(60-70^{\circ} \mathrm{C}\right)$ inactivates the specifier proteins while preserving myrosinase, which increases the yield of SF in broccoli by 3-7fold [47]. Cooking cruciferous vegetables in high heat destroys their myrosinase, and ITCs are also heat labile [48]. However, enteric microflora of both humans and animals possess myrosinase activity and may convert a significant portion of ingested glucosinolates to ITCs [16, 49]. Glucosinolate levels vary greatly among cruciferous vegetables. For example, total glucosinolate contents in garden cress and Pe-tsai Chinese cabbage are 389 and $20 \mathrm{mg}$ per $100 \mathrm{~g}$ fresh weight [50]. The average level of total ITC content ranges from $1.5 \mu \mathrm{mol}$ per $100 \mathrm{~g}$ fresh cauliflower to $61.3 \mu \mathrm{mol}$ per $100 \mathrm{~g}$ mustard greens [51].

\section{Metabolism and Urinary Disposition}

ITCs, upon oral consumption, are primarily metabolized by the mercapturic acid pathway. They first conjugate with glutathione (GSH) via the cysteine sulfhydryl group, which occurs spontaneously but is further enhanced by glutathione $S$ transferase (GST). ITCs are substrates of several isoforms of GSTs, with GSTP1-1 and GSTM1-1 as the most efficient catalysts of ITCs and GSTM4-4 as the least effective [52]. The conjugates undergo multistep enzymatic modifications on the GSH moiety to form cysteinylglycine-, cysteine-, and $\mathrm{N}$-acetylcysteine (NAC) conjugates; these are excreted in the urine (Fig. 1a) [53-61]. The organ sites for ITC metabolism have not been fully investigated, but tissue homogenates of both the liver and kidney catalyze the metabolic conversion [54]. The 1,2-benzenedithiol (BDT)-based cyclocondensation assay [62] was used to measure the absorption and bioavailability of ITCs as well as plasma and urine concentrations of ITC equivalents (ITC plus its metabolites formed in the mercapturic acid pathway) in humans and rodents in many of the studies described below. Plasma ITC levels increased very rapidly after a single ingestion of broccoli sprout extracts (BSE) containing $200 \mu \mathrm{mol}$ of ITCs (mainly SF) per person; concentration of ITC equivalents reached a maximum of $2.3 \mu \mathrm{mol} / \mathrm{L}$ at $1 \mathrm{~h}$ and declined rapidly thereafter (half-life of $1.8 \mathrm{~h}$ ), whereas the $8 \mathrm{~h}$ urinary concentration of ITC equivalent was $117 \mu \mathrm{M}$ [63]. Approximately $70 \%$ of a SF dose was recovered as SF equivalents in $12 \mathrm{~h}$ of urine in human volunteers given BSE orally [64]. Likewise, approximately $70 \%$ of a SF dose was recovered in the urine as SF equivalent within $12 \mathrm{~h}$ following oral administration of SF or SF-containing BSE to F344 rats, and the urinary concentrations of SF equivalents were 2-4 orders of magnitude higher than the corresponding plasma concentrations, respectively $[65 \cdot, 66,67]$. Bladder tissue uptake of SF was also significantly higher than that of other tissues in F344 rats fed with SF [65 ${ }^{\circ}$. About $72 \%$ of the dose was recovered in $24 \mathrm{~h}$ urine as NAC conjugates ( $60 \%$ as NAC conjugate of SF and $12 \%$ as NAC conjugate of $\mathrm{ECN}$ ) after administration of SF at $50 \mathrm{mg} / \mathrm{kg}$ by intraperitoneal injection to male Sprague Dawley rats [56]. Approximately $95 \%$ of the dose was recovered in $24 \mathrm{~h}$ urine as NAC conjugates $(67 \%$ as NAC conjugate of SF and $28 \%$ as NAC conjugate of ECN) after administration of ECN by intraperitoneal injection to male Sprague-Dawley rats [56]. These results not only show extremely high bioavailability of SF and ECN but also show interconversion of SF and $\mathrm{ECN}$ in vivo. NAC conjugate was also the primary metabolite 
a Orally consumed ITCs

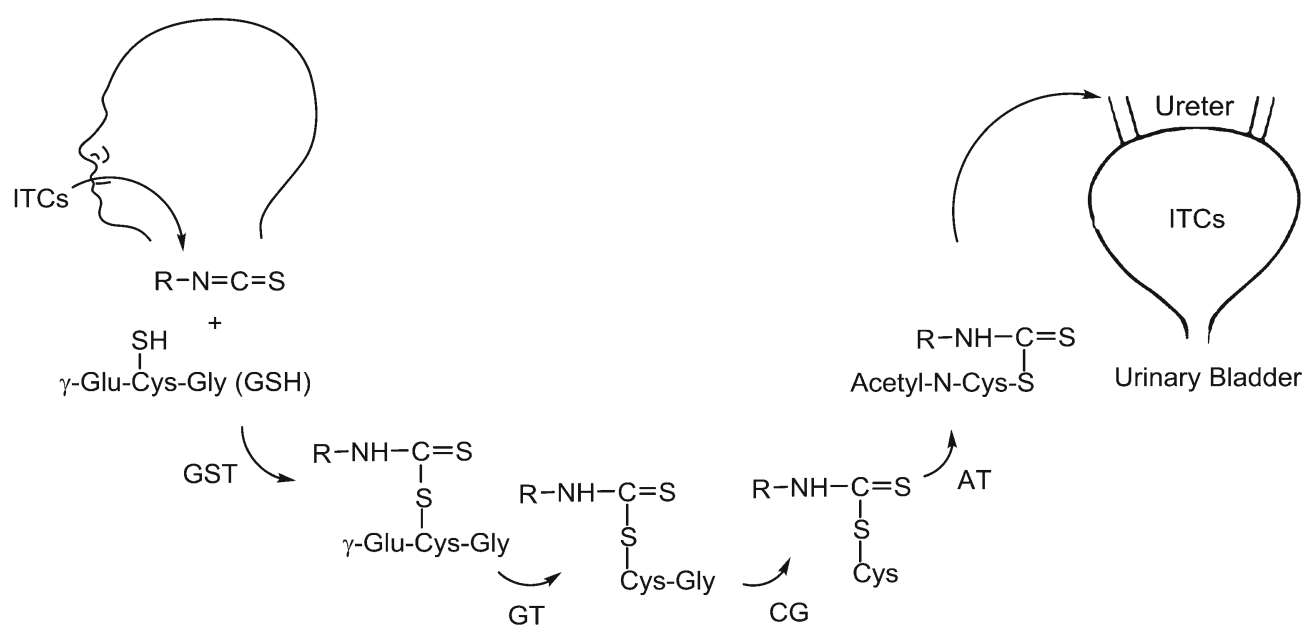

b Modulation of cellular targets by ITCs

$\begin{array}{ll}\uparrow & \text { Caspase-3\&-9 [AITC, BITC, PEITC] } \\ \uparrow & \text { p-Bcl-2 (S-70) [AITC, BITC, SF] } \\ \uparrow & \text { p27 [SF] } \\ \uparrow & \text { JNK [AITC, BITC] } \\ \uparrow & \text { Cyclin B1-Cdk1 complex [SF] }\end{array}$

Fig. 1 Selective delivery of ITCs to the bladder on oral consumption and modulation of cellular targets by ITCs in BC cells and tissues. a ITCs after oral administration are rapidly metabolized via mercapturic acid pathway, and the metabolites which are carriers of ITCs are excreted and concentrated in the urine. GST glutathione- $S$-transferase, $G T \gamma$ -

in overnight urines from human volunteers consuming $400 \mu \mathrm{mol}$ of glucoraphanin (the glucosinolate of SF) [58]. At least $42-54 \%$ of the dose was recovered in the urine as NAC-AITC within 10-12 h of oral administration of 45$90 \mu \mathrm{mol}$ of AITC via horseradish or mustard paste in humans $[61,68]$. In Fischer 344 rats, up to $80 \%$ of orally administered AITC was recovered in $24 \mathrm{~h}$ urine, and tissue levels of AITC in the bladder were approximately 10-fold higher than in other organs [60]. In the same study, in $\mathrm{B}_{6} \mathrm{C} 3 \mathrm{~F}_{1}$ mice, the major metabolite of AITC was thiocyanate ion, suggesting hydrolysis of AITC [60]. When AITC was administered to F344 rats bearing both orthotopic bladder tumors and subcutaneous tumors derived from the same cell line (AY-27 BC cells), tissue levels of AITC equivalent were three orders of magnitude higher in the orthotopic tumors compared to those in subcutaneous tumors, and urinary concentrations of AITC equivalent were also 2-3 orders of magnitude higher than in the blood [32]. Similarly, $54 \%$ of the BITC dose was recovered as NAC-BITC in 10-12 h urine in human volunteers consuming $\downarrow \quad$ Cyclin A, B [AITC, BITC, PEITC, SF]

$\downarrow \quad \mathrm{Cdc} 25 \mathrm{C}[\mathrm{BITC}, \mathrm{SF}]$

$\downarrow \quad \alpha$-tubulin, $\beta$-tubulin [AITC, BITC, PEITC, SF]

$\downarrow \quad$ Survivin [ECN, SF]

$\downarrow \quad$ Cox-2, PGE2 [SF]

$\downarrow \quad$ VEGF [AITC]

$\downarrow \quad$ EGFR [ECN, SF]

glutamyltranspeptidase, $C G$ cysteinylglycinase, $A T N$-acetyltransferase. The organ sites for ITC metabolism have not been fully investigated. b ITCs modulate many proteins that are important for cancer growth and progression

pure BITC (14.4 mg or $96.5 \mu \mathrm{mol})$ or fresh garden cress $(25$ $27 \mathrm{~g}$ ) per person [55]. Also, $65 \%$ of the dose was recovered as NAC conjugate in $12 \mathrm{~h}$ urine in the above study. NAC-ITCs and the other conjugates formed in the mercapturic acid pathway are biologically similar to their parent compounds, as they are carriers of ITCs [69]. The half-time of dissociation of NAC-ITC in an aqueous environment at $\mathrm{pH} 7.4$ and $37^{\circ} \mathrm{C}$ is approximately $1-3 \mathrm{~h}$ [70]. Storage of urine in the bladder likely promotes the dissociation of NAC-ITCs and other ITC metabolites to ITCs. Collectively, orally dosed ITCs are excreted and markedly concentrated in the urine. As the vast majority of $\mathrm{BC}$ arises in the urothelium, dietary ITCs are attractive for the prevention of $\mathrm{BC}$ and $\mathrm{BC}$ recurrence.

\section{Cellular Uptake of ITCs}

AITC, BITC, PEITC, and SF accumulated rapidly in cultured cells with the peak intracellular concentration reached within 
0.5-3 h of treatment, which is 100 - to 200 -fold higher than the extracellular ITC concentration [42]. The ITCs apparently enter cells by diffusion and rapidly conjugate with intracellular thiols, mainly GSH [41]. GSH, abundant in animal and human cells $(0.2-10 \mathrm{mM})$ [71], serves as the major driving force for intracellular accumulation of ITCs [72]. Initial uptake rates of these ITCs correlate well with the non-enzymatic second-order rate constants of their conjugation reactions with GSH [72], and cellular GST enhances ITC uptake by catalyzing ITC conjugation with GSH [72]. ITC accumulation in cells may also cause GSH depletion [42]. ITCs may also bind to proteins via reaction with their cysteine sulfhydryl groups or with an amino group $[41,73]$. ITC binding to cellular proteins may be influenced by its lipophilicity, concentration, and exposure time $[41,42,72]$. ITCs may also modulate cancerrelated proteins via induction of reactive oxygen species [74].

High intracellular accumulation of AITC, BITC, and SF is followed by a rapid export by membrane transporter pumps such as multidrug resistance-associated protein-1 (MRP-1) and P-glycoprotein-1 (Pgp-1) [75, 76]. Interestingly, BITC, PEITC, and other ITCs (10-100 $\mu \mathrm{M})$, but not AITC and SF, inhibit Pgp-1, MRP-1, and breast cancer resistance proteinmediated cellular export of several anticancer agents in cultured cells, which likely results from inhibition of the ATPase of the transporters [77, 78]. This suggests that certain ITCs may help mitigate drug resistance. However, whether export of ITCs is in any sense self-limiting is not known.

\section{Chemopreventive Activities of Cruciferous Vegetables and ITCs in Preclinical Studies}

Our discussion will be focused on the chemopreventive activities of cruciferous vegetables and ITCs that are particularly relevant to the prevention of NMIBC recurrence and progression. Summarized in Table 2 are cell lines and animal models that were employed to evaluate the inhibitory effects of ITCs on $\mathrm{BC}$.

\section{Cell Studies}

ITCs, including AITC, BITC, PEITC, and SF, inhibit the survival and proliferation of a wide array of animal and human $\mathrm{BC}$ cell lines $[16,22,79,80]$. The growth inhibitory activities of ITCs are not specific to a particular cancer cell line, but they appear to inhibit cancer cells more than normal cells. For example, AITC inhibited human UM-UC-3 BC cells and rat AY-27 BC cells with an $\mathrm{IC}_{50}$ of $2.7-3.3 \mu \mathrm{M}$ (72 $\mathrm{h}$ treatment), but it was more than 21 times less effective on normal human bladder epithelial cells [32]. AITC did not elicit significant cell cycle arrest and apoptosis in normal human bladder epithelial cells, even at concentrations that are highly effective against their malignant counterparts [32]. Both SF and ECN were more toxic to human BC cells, including RT4 cells (derived from low-grade non-muscle-invasive cancer), J82 cells, and UM-UC-3 cells (derived from high-grade muscleinvasive cancers) than to normal bladder urothelial cells [31]. Growth inhibitory activities of ITCs are associated with cell cycle arrest, induction of apoptosis, inhibition of angiogenesis, and invasion $[81 \cdot, 82-84,85 \bullet, 86]$. PEITC and SF also inhibit inflammatory response, including downregulation of cyclooxygenase-2 (Cox-2) and reduction of prostaglandin E2 level [87, 88]. Toll-like receptor 4 (TLR4) is closely involved in inflammation, and SF $(10-20 \mu \mathrm{M})$ downregulated TLR4 signaling via thiol-dependent inhibition of its oligomerization in macrophages [89].

\section{Animal Studies}

ITCs have been evaluated in several animal models of BC. In rats treated simultaneously with BITC $(0.001-0.1 \%$ in diet) and $N$-butyl- $N$-(4-hydroxybutyl)nitrosamine (BBN; at $0.005 \%$ in drinking water for 40 weeks), BITC inhibited BBN-induced bladder tumorigenesis in a dose-dependent manner [90]. Dietary BSE (rats consuming total ITCs, mainly SF, at 40 $160 \mu \mathrm{mol} / \mathrm{kg} /$ day) reduced BBN-induced BC incidence by up to $61 \%$ and tumor multiplicity by up to $77 \%$ as well as reducing tumor size and delaying tumor progression [67]. In

Table 2 Inhibitory activities of ITCs against BC cells in vitro and in vivo

\begin{tabular}{lll}
\hline ITCs, vegetables and other agents & BC cell lines/BC animal models & Reference No. \\
\hline AITC, AITC-rich mustard seed powder, NAC-AITC, & Cell lines: UM-UC-3, T24, AY-27 & {$[69,81-83]$} \\
AITC \& celecoxib & Rats: orthotopic AY-27 BC model, subcutaneous AY-27 BC model & {$[32,84,85 \cdot, 91 \cdot]$} \\
BITC & Cell lines: UM-UC-3, T24 & {$[69,82,83]$} \\
& Rats: orthotopic BBN-induced BC model & {$[90]$} \\
PEITC & Cell lines: UM-UC-3, T24 & {$[69,82,83]$} \\
SF, SF-rich broccoli sprout extracts, ECN & Cell lines: UM-UC-3, T24, RT4, J82, UM-UC-3, normal human & {$[69,82,83,95,96,101]$} \\
& urothelial cells, 5637 & {$[67]$} \\
& Rats: orthotopic BBN-induced BC model & {$[31,86]$} \\
\hline
\end{tabular}


rats bearing orthotopic $\mathrm{BC}$, initiated by transurethral inoculation of AY-27 cells, AITC or NAC-AITC at $10 \mu \mathrm{mol} / \mathrm{kg}$ daily by gavage for 3 weeks inhibited tumor growth by $30-40 \%$ and significantly inhibited tumor muscle invasion, while higher AITC doses were not more effective [32, 91•]. In the same animal model, AITC-rich mustard seed powder (MSP-1), given by gavage at the daily AITC dose of $9 \mu \mathrm{mol} / \mathrm{kg}(71.5 \mathrm{mg}$ powder per kilogram), showed antitumor efficacy similar to that of pure AITC, although, as with AITC, a higher MSP-1 dose did not lead to greater effect [84]. Moreover, AITC in combination with celecoxib, a selective Cox-2 inhibitor, led to increased tumor inhibition in the orthotopic BC model mentioned above, compared with each agent used alone [85•]. The combination regime was also more effective than each single agent in inhibiting microvessel formation and stimulating microvessel maturation in tumor tissues [85•].

SF and ECN as well as BSE were evaluated in a subcutaneous xenograft model in mice, initiated by inoculation of UM-UC-3 BC cells. Mice bearing the UM-UC-3 xenografts were treated with SF or ECN $(295 \mu \mathrm{mol} / \mathrm{kg})$ daily by gavage or $2-4 \% \mathrm{BSE}$ in the diet (providing approximately $171-$ $297 \mu \mathrm{mol} \mathrm{SF}$ or its precursor and 25-40 $\mu \mathrm{mol}$ ECN or its precursor per kilogram daily) for 2 weeks; SF, ECN, and BSE significantly inhibited tumor growth by 33-58 \%; this effect was associated with decreased Ki67 staining and accumulation of SF/ECN metabolites in the tumor tissues [31]. When mice bearing subcutaneous UM-UC-3 xenografts were treated with a SF extract of broccoli sprouts $(68 \mu \mathrm{mol}$ SF per kilogram) by gavage twice daily for 5 weeks, tumor growth was inhibited by $63 \%$, which was associated with decreased angiogenesis, and infiltration of eosinophils, basophils, and lymphocytes in the tumors indicative of enhanced immune reaction [86].

Inhibition of BC cells and tumors by the ITCs and ITC-rich vegetable extracts described above is accompanied by modulation of many molecular targets in the cells and tumor tissues. These changes are described in detail in the following section.

\section{Molecular Mechanisms by Which Cruciferous Vegetables and ITCs Target BC Cells}

ITCs modulate a number of proteins involved in the growth and progression of BC (Fig. 1). Many other proteins are modulated by ITCs in other tumor types [24, 92, 93]. In UM-UC-3 cells, AITC, BITC, PEITC, and SF (7.5-30 $\mu \mathrm{M})$ downregulated cyclin $\mathrm{A}$ or cyclin $\mathrm{B} 1$, consistent with cell cycle arrest in $\mathrm{S}$ phase or G2/M phase [82]. MSP-1 and NAC-AITC showed activities similar to those of AITC $[32,84]$. In RT4 cells, both BITC and NAC-BITC downregulated Cdc25C and caused arrest in G2/M and $\mathrm{S}$ phases [69]. SF-rich BSE also arrested UM-UC-3 cells in G2/M and S phases, which was accompanied by downregulation of $\mathrm{Cdc} 25 \mathrm{C}$ and disruption of mitotic spindles [94]. However, SF and ECN (5-20 $\mu \mathrm{M})$ were shown to cause only $\mathrm{G} 2 / \mathrm{M}$ phase arrest in UM-UC-3 cells and other human BC cell lines (RT4 and J82) [31]. In human T24 BC cells, SF caused G0/G1 arrest, which was associated with upregulation of cyclin-dependent kinase (cdk) inhibitor p27 [95]. Moreover, in human 5637 BC cells, SF caused only M phase arrest, which was associated with induction of cyclin B1 and phosphorylation of Cdk1 [96]. Thus, cell cycle arrest in specific phases and modulation of cell cycle regulators by an ITC may vary with experimental conditions and cell lines. Tubulins and microtubules are essential for mitosis. AITC (7.5-30 $\mu \mathrm{M})$ bound to multiple cysteine residues of $\alpha$ tubulin (Cys127, Cys 347, and Cys 376) and $\beta$-tubulin (Cys12, Cys239, Cys303, and Cys354) stimulated their ubiquitination and degradation in UM-UC-3 cells, which was associated with strong mitotic arrest [81•]. NAC-AITC (7.5-15 $\mu \mathrm{M})$ also downregulated $\alpha$ - and $\beta$-tubulins in AY-27 cells [91•]. BITC, PEITC, and SF $(10-20 \mu \mathrm{M})$ bound to cysteine residues of $\alpha$ - and $\beta$-tubulins when tested in human lung cancer cells, including Cys347 of $\alpha$-tubulin and Cys303 of $\beta$ tubulin, which led to proteasomal degradation of the tubulins [97, 98]. BITC, PEITC, and SF also inhibited tubulin polymerization [98, 99], but AITC showed no such effect [81•].

AITC (7.5-30 $\mu \mathrm{M})$ stimulated phosphorylation of B cell lymphoma 2 (Bcl-2) at Ser-70 through activation of c-Jun Nterminal kinases (JNK) in UM-UC-3 cells, which led to induction of apoptosis [81•]. AITC did not modulate other Bcl-2 family proteins, including Bcl-2 homologous antagonist killer (Bak), B cell lymphoma-extra large (Bcl-xl), Bcl-2-associated death promoter (Bad), Bcl-2-associated $\mathrm{X}$ protein (Bax), and BH3-interacting domain death agonist (Bid) in UM-UC-3 cells, but it disrupted the association of Bcl-2 with Bax and induced mitochondria-mediated apoptosis, including the loss of mitochondrial transmembrane potential, cytoplasmic accumulation of cytochrome $C$, activation of caspases $9 / 3$, and cleavage of poly(ADP-ribose)polymerase (PARP) and lamin B1 [81•]. MSP-1 and NAC-AITC also stimulated apoptosis in UM-UC-3 cells and AY-27 cells [32, 84, 91•]. AITC-induced apoptosis depends on mitotic arrest, and Bcl-2 phosphorylation is the molecular link between mitotic arrest and apoptosis in AITC-treated cells [81•]. Bcl-2 phosphorylation also increased in UM-UC-3 cells after treatment with BITC and PEITC (7.5 $\mu \mathrm{M})$ [83]. BITC stimulated JNK-dependent Bcl2 phosphorylation, when tested in human $\mathrm{T}$ cell leukemia Jurkat cells [100]. Mitochondria play a key role in ITCinduced apoptosis. Besides Bcl-2 phosphorylation mentioned above, the apoptosis-inducing potentials of AITC, BITC, PEITC, and SF in UM-UC-3 cells correlate well with their ability to modulate mitochondria; they induce mitochondria translocation of Bak, disrupt the association of Bcl-xl with Bak and Bax in mitochondrial membrane, and cause the collapse of mitochondrial transmembrane potential and release of cytochrome C [83]. AITC-induced apoptosis in UM-UC-3 
cells can be blocked by either a Bcl-2 mutant that does not respond to AITC or a caspase 9 inhibitor [81•]. Reactive oxygen species have also been implicated in apoptosis induction by ITCs. In human T24 BC cells, induction of apoptosis by SF is linked to a reactive oxygen species-mediated mitochondrial apoptosis pathway [101].

Many other proteins involved in apoptosis are also modulated by ITCs. Cox-2, a pro-inflammatory oncogene that plays a pivotal role in $\mathrm{BC}$ development, is frequently overexpressed in $\mathrm{BC}$ and is associated with stage, grade, recurrence, and prognosis of BC [102]. SF (5-20 $\mu \mathrm{M})$ downregulated Cox-2 in T24 cells via activation of p38 and inhibition of NF-kB binding to the Cox-2 promoter [88, 103]. AITC does not modulate Cox-2, but AITC in combination with celecoxib, a selective Cox-2 inhibitor, was more effective than each agent used alone for inhibition of orthotopic $\mathrm{BC}$ growth and muscle invasion in rats [85•]. This finding suggests that SF may complement AITC for BC inhibition. Vascular endothelial growth factor (VEGF) stimulates cancer angiogenesis, invasion, and metastasis. MSP-1 (13-26 $\mu \mathrm{M}$ of AITC) and NAC-AITC (7.5-15 $\mu \mathrm{M})$ caused significant downregulation of VEGF in both UM-UC-3 cells and AY-27 cells [84, 91•]. In RT4 cells and UM-UC-3 cells, SF and ECN (5-20 $\mu \mathrm{M})$ also downregulated survivin and epidermal growth factor receptor (EGFR) [31].

In vivo, inhibition of orthotopic BC in rats by AITC (10 $\mu \mathrm{mol} / \mathrm{kg}$ daily), MSP-1 (providing AITC at $9 \mu \mathrm{mol} / \mathrm{kg}$ daily), or NAC-AITC (10 $\mu \mathrm{mol} / \mathrm{kg}$ daily), as described in the animal studies section above, was associated with significant downregulation of cyclin B1, $\alpha$-/ $\beta$-tubulins, and VEGF, and activation of caspase 3 in the tumor tissues [32, 84, 91 •]. Significant inhibition of subcutaneous UM-UC-3 tumors in athymic mice by SF ( $68 \mu \mathrm{mol} / \mathrm{kg}$ twice a day for 5 weeks) was associated with activation of caspase 3 , cytochrome $\mathrm{C}$ release, and downregulation of survivin [86]. The SF-treated mice also had tumors with reduced number of blood vessels [86].

\section{Epidemiological Evidence of Chemopreventive Activities of Cruciferous Vegetables in the Bladder}

Several epidemiological studies have shown that intake of cruciferous vegetables is inversely associated with $\mathrm{BC}$ risk. In a case-control study involving $275 \mathrm{BC}$ patients and 825 controls, significant inverse association between $\mathrm{BC}$ risk and consumption of raw cruciferous vegetables, including broccoli, cabbage, and cauliflower, was observed [adjusted odds ratio (OR) for high ( $\geq 1$ serving/month) versus low ( $<1$ serving/ month) category is $0.64 ; 95 \%$ confidence interval (CI), 0.42-0.97] [104]. In a prospective study involving 47,909 men over 10 years, cruciferous vegetable consumption was inversely associated with $\mathrm{BC}$ risk [relative risk $(\mathrm{RR})=0.49$; $95 \%$ CI, 0.32-0.75] [105]. A meta-analysis of five cohorts and five case-control studies also showed significantly decreased risk of $\mathrm{BC}$ with increased intake of cruciferous vegetables $[\mathrm{RR}=0.80 ; 95 \% \mathrm{CI}, 0.69-0.92)[106 \bullet]$. However, no study has directly examined the association of cruciferous vegetable intake and $\mathrm{BC}$ recurrence. In a retrospective cohort of $239 \mathrm{BC}$ patients, broccoli intake, in particular raw broccoli, at least one serving per month, was significantly associated with reduced overall mortality [hazard ratio (HR), 0.57; $95 \%$ CI, 0.39-0.83] and BC-specific mortality (HR, $0.43 ; 95 \%$ CI, $0.25-0.74$ ) [107]. These epidemiological findings are consistent with results from cell and animal studies discussed before and provide additional evidence that ITC intake may slow BC growth, progression, and recurrence.

\section{Potential Toxicity of ITCs}

Several studies have shown adverse effects of certain ITCs in animals. F344 rats (male and female) consuming a diet containing $0.1 \%$ of BITC, PEITC, or an analog for 1-14 days showed acute toxicity in the bladder, such as hemorrhage, inflammation, and necrosis, followed by gradual increase in the thickness of the bladder epithelium, and papillary or nodular hyperplasia [108]. Male F344 rats treated with BITC at $0.1 \%$ in the diet for 40 weeks alone showed epithelial hyperplasia, although BITC strongly prevented BBN from inducing dysplasia, papilloma, or carcinoma [90]. F344/DuCrj rats (male and female) upon drinking water containing AITC or an AITC-rich horseradish extract (estimated AITC intake 30$40 \mathrm{mg} / \mathrm{kg} /$ day) for 13 weeks developed simple hyperplasia $(100 \%)$ and papillary or nodular hyperplasia (20-40\%) [109]. In another study, administration of AITC by gavage $(12-25 \mathrm{mg} / \mathrm{kg}$ or $121-252 \mu \mathrm{mol} / \mathrm{kg}$ ) daily 5 days per week for 103 weeks induced transitional cell papillomas (prevalence 4-8 \%) in male F344 rats but not female rats, which was suspected to be due to higher urinary concentrations of ITC metabolites in the male rats [110]. In contrast, AITC given to F344 rats orally at $10 \mu \mathrm{mol} / \mathrm{kg}$ daily inhibited orthotopic BC, while higher AITC doses were not more effective [32]. PEITC induced bladder toxicities only when its dietary level exceeded $0.01-0.05 \%$ (approximately $37-183 \mu \mathrm{mol} / \mathrm{kg}$ daily) for 32 weeks [111]. Given that orally consumed ITCs are concentrated in the urine and ITCs are highly electrophilic, the bladder damage discussed above may be mainly due to overdosing. It is important to note that the toxicities observed in animal studies result from doses that are several orders of magnitude greater than those observed in human epidemiologic studies [112].

\section{Concluding Remarks}

Accumulating evidence, from both epidemiologic and preclinical studies, suggests that naturally occurring ITCs as well as 
ITC-containing cruciferous vegetables and vegetable extracts may be useful for prevention of $\mathrm{BC}$ development and recurrence in humans. ITCs can be administered orally but are selectively delivered to the bladder via urinary excretion, contrasting with currently available intravesical agents that are delivered via a urethral catheter. Further studies are needed, in order to better understand the chemopreventive activity of ITCs and their mechanisms of actions, to identify biomarkers potentially useful for clinical evaluation of ITCs in patients, and to evaluate the chemopreventive efficacy of mechanismbased combination of multiple ITCs or ITC with other agents. Clearer understanding of the potential adverse effects of these compounds in humans is also needed. Additional population studies may further clarify the relationship between consumption of cruciferous vegetables and $\mathrm{BC}$ risk, risk of $\mathrm{BC}$ recurrence, and survival of $\mathrm{BC}$ patients. Rodent models may understate the cancer chemopreventive activities of ITCs in the bladder, as rodents are prone to frequent urination, whereas urine storage in the bladder in humans may facilitate the dissociation of ITC metabolites and increase the exposure of BC cells to ITCs in the urine. For this reason, the available preclinical and epidemiologic data suggest a need for clinical evaluation of ITCs or ITC-containing vegetable extracts in $\mathrm{BC}$ patients.

Acknowledgments This work was supported in part by US NIH/NCI grants R01CA164574 and R01CA172855.

\section{Compliance with Ethics Guidelines}

Conflict of Interest Omkara L. Veeranki, Arup Bhattacharya, Li Tang, James R. Marshall, and Yuesheng Zhang declare that they have no conflict of interest.

Human and Animal Rights and Informed Consent This article does not contain any new studies with human or animal subjects performed by any of the authors.

\section{References}

Papers of particular interest, published recently, have been highlighted as:

- Of importance

1. Siegel R, Ma J, Zou Z, et al. Cancer statistics, 2014. CA Cancer J Clin. 2014;64(1):9-29.

2. Freedman ND, Silverman DT, Hollenbeck AR, et al. Association between smoking and risk of bladder cancer among men and women. JAMA. 2011;306(7):737-45.

3. Burger M, Catto JW, Dalbagni G, et al. Epidemiology and risk factors of urothelial bladder cancer. Eur Urol. 2013;63(2):234-41.

4. Juffs HG, Moore MJ, Tannock IF. The role of systemic chemotherapy in the management of muscle-invasive bladder cancer. Lancet Oncol. 2002;3(12):738-47.
5. Babjuk M, Burger M, Zigeuner R, et al. EAU guidelines on nonmuscle-invasive urothelial carcinoma of the bladder: update 2013. Eur Urol. 2013;64(4):639-53.

6. Metts MC, Metts JC, Milito SJ, et al. Bladder cancer: a review of diagnosis and management. J Natl Med Assoc. 2000;92(6): 285-94.

7. Heney N, Proppe K, Prout Jr G, et al. Invasive bladder cancer: tumor configuration, lymphatic invasion and survival. J Urol. 1983;130(5):895-7.

8. Sylvester R, van der Meijden A, Oosterlinck W, et al. Predicting recurrence and progression in individual patients with stage Ta T1 bladder cancer using EORTC risk tables: a combined analysis of 2596 patients from seven EORTC trials. Eur Urol. 2006;49(3): 466-77.

9. Kondas J, Kiss L, Hatar A, et al. The effect of intravesical mitomycin $\mathrm{C}$ on the recurrence of superficial (Ta-T1) bladder cancer. A Hungarian Multicenter Study. Int Urol Nephrol. 1999;31(4): 451-6.

10. Knowles MA. Molecular subtypes of bladder cancer: Jekyll and Hyde or chalk and cheese? Carcinogenesis. 2006;27(3):361-73.

11. Wu XR. Urothelial tumorigenesis: a tale of divergent pathways. Nat Rev Cancer. 2005;5(9):713-25.

12. Botteman MF, Pashos CL, Redaelli A, et al. The health economics of bladder cancer: a comprehensive review of the published literature. Pharmacoeconomics. 2003;21(18):1315-30.

13. Jacobs BL, Lee CT, Montie JE. Bladder cancer in 2010: how far have we come? CA Cancer J Clin. 2010;60(4):244-72.

14. O'Donnell MA, Boehle A. Treatment options for BCG failures. World J Urol. 2006;24(5):481-7.

15. Hilton WM, Ercole B, Parekh DJ, et al. Efficacy of combined intravesical immunotherapy and chemotherapy for non-muscle invasive bladder cancer. Expert Rev Anticancer Ther. 2011;11(6): 949-57.

16. Zhang Y. Allyl isothiocyanate as a cancer chemopreventive phytochemical. Mol Nutr Food Res. 2010;54(1):127-35.

17. Nakamura Y, Kawakami M, Yoshihiro A, et al. Involvement of the mitochondrial death pathway in chemopreventive benzyl isothiocyanate-induced apoptosis. J Biol Chem. 2002;277(10): 8492-9.

18. Melchini A, Costa C, Traka M, et al. Erucin, a new promising cancer chemopreventive agent from rocket salads, shows antiproliferative activity on human lung carcinoma A549 cells. Food Chem Toxicol. 2009;47(7):1430-6.

19. Aras U, Gandhi YA, Masso-Welch PA, et al. Chemopreventive and anti-angiogenic effects of dietary phenethyl isothiocyanate in an N-methyl nitrosourea-induced breast cancer animal model. Biopharm Drug Dispos. 2013;34(2):98-106.

20. Cheung KL, Kong AN. Molecular targets of dietary phenethyl isothiocyanate and sulforaphane for cancer chemoprevention. AAPS J. 2010;12(1):87-97.

21. Zhang Y, Tang L. Discovery and development of sulforaphane as a cancer chemopreventive phytochemical. Acta Pharmacol Sin. 2007;28(9):1343-54.

22. Juge N, Mithen RF, Traka M. Molecular basis for chemoprevention by sulforaphane: a comprehensive review. Cell Mol Life Sci. 2007;64(9):1105-27.

23. Higdon JV, Delage B, Williams DE, et al. Cruciferous vegetables and human cancer risk: epidemiologic evidence and mechanistic basis. Pharmacol Res. 2007;55(3):224-36.

24. Kim SH, Sehrawat A, Singh SV. Dietary chemopreventative benzyl isothiocyanate inhibits breast cancer stem cells in vitro and in vivo. Cancer Prev Res. 2013;6(8):782-90.

25. Wang D, Upadhyaya B, Liu Y, et al. Phenethyl isothiocyanate upregulates death receptors 4 and 5 and inhibits proliferation in human cancer stem-like cells. BMC Cancer. 2014;14(1):591-603. 
26. Li Y, Zhang T. Targeting cancer stem cells with sulforaphane, a dietary component from broccoli and broccoli sprouts. Future Oncol. 2013;9(8):1097-103.

27. Li SH, Fu J, Watkins DN, et al. Sulforaphane regulates self-renewal of pancreatic cancer stem cells through the modulation of Sonic hedgehog-GLI pathway. Mol Cell Biochem. 2013;373(1-2): 217-27.

28. Hecht SS. Chemoprevention by isothiocyanates. J Cell Biochem Suppl. 1995;22(1):195-209.

29. Dinkova-Kostova AT. Chemoprotection against cancer by isothiocyanates: a focus on the animal models and the protective mechanisms. Top Curr Chem. 2013;329(1):179-201.

30. Hecht SS. Inhibition of carcinogenesis by isothiocyanates. Drug Metab Rev. 2000;32(3-4):395-411.

31. Abbaoui B, Riedl KM, Ralston RA, et al. Inhibition of bladder cancer by broccoli isothiocyanates sulforaphane and erucin: characterization, metabolism, and interconversion. Mol Nutr Food Res. 2012;56(11):1675-87.

32. Bhattacharya A, Tang L, Li Y, et al. Inhibition of bladder cancer development by allyl isothiocyanate. Carcinogenesis. 2010;31(2): 281-6.

33. Sehrawat A, Singh SV. Benzyl isothiocyanate inhibits epithelialmesenchymal transition in cultured and xenografted human breast cancer cells. Cancer Prev Res. 2011;4(7):1107-17.

34. Warin R, Xiao D, Arlotti JA, et al. Inhibition of human breast cancer xenograft growth by cruciferous vegetable constituent benzyl isothiocyanate. Mol Carcinog. 2010;49(5):500-7.

35. Matsui TA, Murata H, Sakabe T, et al. Sulforaphane induces cell cycle arrest and apoptosis in murine osteosarcoma cells in vitro and inhibits tumor growth in vivo. Oncol Rep. 2007;18(5): 1263-8.

36. Boreddy SR, Pramanik KC, Srivastava SK. Pancreatic tumor suppression by benzyl isothiocyanate is associated with inhibition of PI3K/AKT/FOXO pathway. Clin Cancer Res. 2011;17(7): 1784-95.

37. Singh AV, Xiao D, Lew KL, et al. Sulforaphane induces caspasemediated apoptosis in cultured PC-3 human prostate cancer cells and retards growth of PC-3 xenografts in vivo. Carcinogenesis. 2004;25(1):83-90.

38. Singh SV, Warin R, Xiao D, et al. Sulforaphane inhibits prostate carcinogenesis and pulmonary metastasis in TRAMP mice in association with increased cytotoxicity of natural killer cells. Cancer Res. 2009;69(5):2117-25.

39. Khor TO, Cheung WK, Prawan A, et al. Chemoprevention of familial adenomatous polyposis in Apc(Min/+) mice by phenethyl isothiocyanate (PEITC). Mol Carcinog. 2008;47(5):321-5.

40. Myzak MC, Dashwood WM, Orner GA, et al. Sulforaphane inhibits histone deacetylase in vivo and suppresses tumorigenesis in Apc-minus mice. FASEB J. 2006;20(3):506-8.

41. Zhang Y. The molecular basis that unifies the metabolism, cellular uptake and chemopreventive activities of dietary isothiocyanates. Carcinogenesis. 2012;33(1):2-9.

42. Zhang Y. Role of glutathione in the accumulation of anticarcinogenic isothiocyanates and their glutathione conjugates by murine hepatoma cells. Carcinogenesis. 2000;21(6):1175-82.

43. Jiao D, Eklind KI, Choi CI, et al. Structure-activity relationships of isothiocyanates as mechanism-based inhibitors of 4(methylnitrosamino)-1-(3-pyridyl)-1-butanone-induced lung tumorigenesis in A/J mice. Cancer Res. 1994;54(16):4327-33.

44. Bones AM, Rossiter JT. The myrosinase-glucosinolate system, its organisation and biochemistry. Physiol Plant. 1996;97(1): 194-208.

45. Bernardi R, Negri A, Ronchi S, et al. Isolation of the epithiospecifier protein from oil-rape (Brassica napus ssp. oleifera) seed and its characterization. FEBS Lett. 2000;467(2-3):296-8.
46. Fenwick GR, Heaney RK. Glucosinolates and their breakdown products in cruciferous crops, foods, and feeding-stuffs. Food Chem. 1983;11(4):249-71.

47. Matusheski NV, Juvik JA, Jeffery EH. Heating decreases epithiospecifier protein activity and increases sulforaphane formation in broccoli. Phytochemistry. 2004;65(9):1273-81.

48. Rouzaud G, Young SA, Duncan AJ. Hydrolysis of glucosinolates to isothiocyanates after ingestion of raw or microwaved cabbage by human volunteers. Cancer Epidemiol Biomarkers Prev. 2004;13(1):125-31.

49. Zhang Y. Cancer-preventive isothiocyanates: Measurement of human exposure and mechanism of action. Mutat Res. 2004;555(1-2):173-90.

50. McNaughton SA, Marks GC. Development of a food composition database for the estimation of dietary intakes of glucosinolates, the biologically active constituents of cruciferous vegetables. $\mathrm{Br} \mathbf{J}$ Nutr. 2003;90(3):687-97.

51. Tang L, Paonessa JD, Zhang Y, et al. Total isothiocyanate yield from raw cruciferous vegetables commonly consumed in the United States. J Funct Foods. 2013;5(4):1996-2001.

52. Kolm RH, Danielson UH, Zhang Y, et al. Isothiocyanates as substrates for human glutathione transferases: structure-activity studies. Biochem J. 1995;311(2):453-9.

53. Chung FL, Morse MA, Eklind KI, et al. Quantitation of human uptake of the anticarcinogen phenethyl isothiocyanate after a watercress meal. Cancer Epidemiol Biomarkers Prev. 1992;1(5): 383-8.

54. Brusewitz G, Cameron BD, Chasseaud LF, et al. The metabolism of benzyl isothiocyanate and its cysteine conjugate. Biochem J. 1977;162(1):99-107.

55. Mennicke WH, Gorler K, Krumbiegel G, et al. Studies on the metabolism and excretion of benzyl isothiocyanate in man. Xenobiotica. 1988;18(4):441-7.

56. Kassahun K, Davis M, Hu P, et al. Biotransformation of the naturally occurring isothiocyanate sulforaphane in the rat: identification of phase i metabolites and glutathione conjugates. Chem Res Toxicol. 1997;10(11):1228-33.

57. Gasper AV, Al-Janobi A, Smith JA, et al. Glutathione Stransferase M1 polymorphism and metabolism of sulforaphane from standard and high-glucosinolate broccoli. Am J Clin Nutr. 2005;82(6):1283-91.

58. Egner PA, Kensler TW, Chen JG, et al. Quantification of sulforaphane mercapturic acid pathway conjugates in human urine by high-performance liquid chromatography and isotope-dilution tandem mass spectrometry. Chem Res Toxicol. 2008;21(10): 1991-6.

59. Ioannou YM, Burka LT, Matthews HB. Allyl isothiocyanate: comparative disposition in rats and mice. Toxicol Appl Pharmacol. 1984;75(2):173-81.

60. Bollard M, Stribbling S, Mitchell S, et al. The disposition of allyl isothiocyanate in the rat and mouse. Food Chem Toxicol. 1997;35(10-11):933-43.

61. Jiao D, Ho CT, Foiles $\mathrm{P}$, et al. Identification and quantification of the $\mathrm{N}$-acetylcysteine conjugate of allyl isothiocyanate in human urine after ingestion of mustard. Cancer Epidemiol Biomarkers Prev. 1994;3(6):487-92.

62. Zhang Y. The 1,2-benzenedithiole-based cyclocondensation assay: a valuable tool for the measurement of chemopreventive isothiocyanates. Crit Rev Food Sci Nutr. 2012;52(6):525-32.

63. Ye L, Dinkova-Kostova AT, Wade KL, et al. Quantitative determination of dithiocarbamates in human plasma, serum, erythrocytes and urine: pharmacokinetics of broccoli sprout isothiocyanates in humans. Clin Chim Acta. 2002;316(1-2):43-53.

64. Egner PA, Chen JG, Wang JB, et al. Bioavailability of sulforaphane from two broccoli sprout beverages: results of a short-term, 
cross-over clinical trial in Qidong, China. Cancer Prev Res. 2011;4(3):384-95.

65. Veeranki OL, Bhattacharya A, Marshall JR, et al. Organ-specific exposure and response to sulforaphane, a key chemopreventive ingredient in broccoli: implications for cancer prevention. $\mathrm{Br} \mathrm{J}$ Nutr. 2012;2(109):25-32. This study shows that SF is rapidly eliminated through urinary excretion and that $S F$ reaches different organs in vastly different concentrations. Hence, it provides a basis for prioritising organs for further chemopreventive study of $S F$ and suggests that $S F$ is highly promising cancer chemopreventive agent in the bladder.

66. Zhang Y, Munday R, Jobson HE, et al. Induction of GST and NQO1 in cultured bladder cells and in the urinary bladders of rats by an extract of broccoli (Brassica oleracea italica) sprouts. J Agric Food Chem. 2006;54(25):9370-6.

67. Munday R, Mhawech-Fauceglia P, Munday CM, et al. Inhibition of urinary bladder carcinogenesis by broccoli sprouts. Cancer Res. 2008;68(5):1593-600.

68. Shapiro TA, Fahey JW, Wade KL, et al. Human metabolism and excretion of cancer chemoprotective glucosinolates and isothiocyanates of cruciferous vegetables. Cancer Epidemiol Biomarkers Prev. 1998;7(12):1091-100.

69. Tang L, Li G, Song L, et al. The principal urinary metabolites of dietary isothiocyanates, $\mathrm{N}$-acetylcysteine conjugates, elicit the same anti-proliferative response as their parent compounds in human bladder cancer cells. Anticancer Drugs. 2006;17(3):297-305.

70. Conaway CC, Krzeminski J, Amin S, et al. Decomposition rates of isothiocyanate conjugates determine their activity as inhibitors of cytochrome p450 enzymes. Chem Res Toxicol. 2001;14(9): $1170-6$.

71. Anderson ME. Glutathione: an overview of biosynthesis and modulation. Chem Biol Interact. 1998;111(1):1-14.

72. Zhang Y. Molecular mechanism of rapid cellular accumulation of anticarcinogenic isothiocyanates. Carcinogenesis. 2001;22(3): 425-31.

73. Podhradsky D, Drobnica L, Kristian P. Reactions of cysteine, its derivatives, glutathione coenzyme A, and dihydrolipoic acid with isothiocyanates. Experientia. 1979;35(2):154-5.

74. Zhang Y, Li J, Tang L. Cancer-preventive isothiocyanates: dichotomous modulators of oxidative stress. Free Radic Biol Med. 2005;38(1):70-7.

75. Zhang Y, Callaway EC. High cellular accumulation of sulphoraphane, a dietary anticarcinogen, is followed by rapid transporter-mediated export as a glutathione conjugate. Biochem J. 2002;364(Pt 1):301-7.

76. Callaway EC, Zhang Y, Chew W, et al. Cellular accumulation of dietary anticarcinogenic isothiocyanates is followed by transporter-mediated export as dithiocarbamates. Cancer Lett. 2004;204(1):23-31.

77. Ji Y, Morris ME. Membrane transport of dietary phenethyl isothiocyanate by ABCG2 (breast cancer resistance protein). Mol Pharm. 2005;2(5):414-9.

78. Tseng E, Kamath A, Morris ME. Effect of organic isothiocyanates on the P-glycoprotein- and MRP1-mediated transport of daunomycin and vinblastine. Pharm Res. 2002;19(10):1509-15.

79. Singh SV, Singh K. Cancer chemoprevention with dietary isothiocyanates mature for clinical translational research. Carcinogenesis. 2012;33(10):1833-42.

80. Zhang Y, Tang L, Gonzalez V. Selected isothiocyanates rapidly induce growth inhibition of cancer cells. Mol Cancer Ther. 2003;2(10):1045-52.

81. Geng F, Tang L, Li Y, et al. Allyl isothiocyanate arrests cancer cells in mitosis, and mitotic arrest in turn leads to apoptosis via Bcl-2 protein phosphorylation. J Biol Chem. 2011;286(37):32259-67. This article provides a novel anticancer mechanism by which AITC binds to cysteine residues of $\alpha$ - and $\beta$-tubulins, promoting its ubiquitination and degradation, and causing mitotic catastrophe through mitochondrion-mediated apoptosis. Bcl-2 phosphorylation by JNK activation is the molecular link between mitotic arrest and apoptosis in AITC treated UM-UC-3 BC cells.

82. Tang L, Zhang Y. Dietary isothiocyanates inhibit the growth of human bladder carcinoma cells. J Nutr. 2004;134(8):2004-10.

83. Tang L, Zhang Y. Mitochondria are the primary target in isothiocyanate-induced apoptosis in human bladder cancer cells. Mol Cancer Ther. 2005;4(8):1250-9.

84. Bhattacharya A, Li Y, Wade KL, et al. Allyl isothiocyanate-rich mustard seed powder inhibits bladder cancer growth and muscle invasion. Carcinogenesis. 2010;31(12):2105-10.

85. Bhattacharya A, Li Y, Shi Y, et al. Enhanced inhibition of urinary bladder cancer growth and muscle invasion by allyl isothiocyanate and celecoxib in combination. Carcinogenesis. 2013;34(11): 2593-9. This study indicates that AITC together with pharmacologic inhibition of Cox-2 leads to enhanced anticancer efficacy and suggests that SF, which down regulates Cox-2, may complement AITC for inhibition of BC.

86. Wang F, Shan Y. Sulforaphane retards the growth of UM-UC-3 xenographs, induces apoptosis, and reduces survivin in athymic mice. Nutr Res. 2012;32(5):374-80.

87. Lee YM, Cho HJ, Ponnuraj SP, et al. Phenethyl isothiocyanate inhibits 12-O-tetradecanoylphorbol-13-acetate-induced inflammatory responses in mouse skin. J Med Food. 2011;14(4):377-85.

88. Shan Y, Wu K, Wang W, et al. Sulforaphane down-regulates COX-2 expression by activating p38 and inhibiting NF-kappaBDNA-binding activity in human bladder T24 cells. Int J Oncol. 2009;34(4):1129-34.

89. Youn HS, Kim YS, Park ZY, et al. Sulforaphane suppresses oligomerization of TLR4 in a thiol-dependent manner. J Immunol. 2010;184(1):411-9.

90. Okazaki K, Yamagishi M, Son HY, et al. Simultaneous treatment with benzyl isothiocyanate, a strong bladder promoter, inhibits rat urinary bladder carcinogenesis by N-butyl-N-(4hydroxybutyl)nitrosamine. Nutr Cancer. 2002;42(2):211-6.

91. Bhattacharya A, Li Y, Geng F, Munday R, Zhang Y. The principal urinary metabolite of allyl isothiocyanate, N-acetyl-S-(nallylthiocarbamoyl)cysteine, inhibits the growth and muscle invasion of bladder cancer. Carcinogenesis. 2012;33(2):394-8. This study shows that the chemopreventive activity of the principal urinary metabolite of AITC, NAC-AITC, is similar to that of AITC in a preclinical orthotopical BC model; this provides further evidence of the chemopreventive activity of AITC in the bladder, as NAC-AITC is known to serve as a carrier of AITC.

92. Nishikawa A, Furukawa F, Uneyama C, et al. Chemopreventive effects of phenethyl isothiocyanate on lung and pancreatic tumorigenesis in N-nitrosobis(2-oxopropyl)amine-treated hamsters. Carcinogenesis. 1996;17(6):1381-4.

93. Kanematsu S, Yoshizawa K, Uehara N, et al. Sulforaphane inhibits the growth of KPL-1 human breast cancer cells in vitro and suppresses the growth and metastasis of orthotopically transplanted KPL-1 cells in female athymic mice. Oncol Rep. 2011;26(3): 603-8.

94. Tang L, Zhang Y, Jobson HE, Li J, et al. Potent activation of mitochondria-mediated apoptosis and arrest in $\mathrm{s}$ and $\mathrm{m}$ phases of cancer cells by a broccoli sprout extract. Mol Cancer Ther. 2006;5(4):935-44.

95. Shan Y, Sun C, Zhao X, et al. Effect of sulforaphane on cell growth, $\mathrm{G}(0) / \mathrm{G}(1)$ phase cell progression and apoptosis in human bladder cancer T24 cells. Int J Oncol. 2006;29(4):883-8.

96. Park HS, Han MH, Kim GY, et al. Sulforaphane induces reactive oxygen species-mediated mitotic arrest and subsequent apoptosis in human bladder cancer 5637 cells. Food Chem Toxicol. 2014;64(1):157-65. 
97. Mi L, Gan N, Cheema A, et al. Cancer preventive isothiocyanates induce selective degradation of cellular alpha- and beta-tubulins by proteasomes. J Biol Chem. 2009;284(25):17039-51.

98. Mi L, Xiao Z, Hood BL, et al. Covalent binding to tubulin by isothiocyanates. A mechanism of cell growth arrest and apoptosis. J Biol Chem. 2008;283(32):22136-46.

99. Jackson SJ, Singletary KW. Sulforaphane inhibits human MCF-7 mammary cancer cell mitotic progression and tubulin polymerization. J Nutr. 2004;134(9):2229-36.

100. Miyoshi N, Uchida K, Osawa T, et al. A link between benzyl isothiocyanate-induced cell cycle arrest and apoptosis: involvement of mitogen-activated protein kinases in the Bcl-2 phosphorylation. Cancer Res. 2004;64(6):2134-42.

101. Jo GH, Kim GY, Kim WJ, et al. Sulforaphane induces apoptosis in $\mathrm{T} 24$ human urinary bladder cancer cells through a reactive oxygen species-mediated mitochondrial pathway: the involvement of endoplasmic reticulum stress and the Nrf2 signaling pathway. Int J Oncol. 2014;45(4):1497-506.

102. Kim SI, Kwon SM, Kim YS, et al. Association of cyclooxygenase2 expression with prognosis of stage T1 grade 3 bladder cancer. Urology. 2002;60(5):816-21.

103. Shan Y, Wang X, Wang W, et al. p38 MAPK plays a distinct role in sulforaphane-induced up-regulation of ARE-dependent enzymes and down-regulation of COX-2 in human bladder cancer cells. Oncol Rep. 2010;23(4):1133-8.

104. Tang L, Zirpoli GR, Guru K, et al. Consumption of raw cruciferous vegetables is inversely associated with bladder cancer risk. Cancer Epidemiol Biomarkers Prev. 2008;17(4):938-44.
105. Michaud DS, Spiegelman D, Clinton SK, et al. Fruit and vegetable intake and incidence of bladder cancer in a male prospective cohort. J Natl Cancer Inst. 1999;91(7):605-13.

106. Liu B, Mao Q, Lin Y, Zhou F, Xie L. The association of cruciferous vegetables intake and risk of bladder cancer: a meta-analysis. World J Urol. 2013;31(1):127-33. This meta-analysis of five cohorts and five case-control studies evaluated the relationship between cruciferous vegetables intake and risk of BC. It shows that high consumption of cruciferous vegetables is related to decreased risk of $B C$.

107. Tang L, Zirpoli GR, Guru K, et al. Intake of cruciferous vegetables modifies bladder cancer survival. Cancer Epidemiol Biomarkers Prev. 2010;19(7):1806-11.

108. Akagi K, Sano M, Ogawa K, et al. Involvement of toxicity as an early event in urinary bladder carcinogenesis induced by phenethyl isothiocyanate, benzyl isothiocyanate, and analogues in F344 rats. Toxicol Pathol. 2003;31(4):388-96.

109. Hasumura M, Imai T, Cho Y-M, et al. Toxic effects of a horseradish extract and allyl isothiocyanate in the urinary bladder after 13week administration in drinking water to F344 rats. J Toxicol Sci. 2011;36(6):763-74.

110. Dunnick JK, Prejean JD, Haseman J, et al. Carcinogenesis bioassay of allyl isothiocyanate. Fundam Appl Toxicol. 1982;2(3):114-20

111. Ogawa K, Hirose M, Sugiura S, et al. Dose-dependent promotion by phenylethyl isothiocyanate, a known chemopreventer, of twostage rat urinary bladder and liver carcinogenesis. Nutr Cancer. 2001;40(2):134-9.

112. NAS/NRC: Poundage update of food chemicals. NAS/NRC 1982. 